\title{
Experiences Of Individuals Concerning Combined Orthodontic and Orthognathic Surgical Treatment: A Qualitative Twitter Analysis
}

\author{
Guy D. Watts ${ }^{a}$ Panagiotis Christou $^{\mathrm{b}}$ Gregory S. Antonarakis ${ }^{\mathrm{c}}$ \\ ${ }^{a}$ Department of Plastic and Reconstructive Surgery, Sir Charles Gairdner Hospital, Perth, WA, Australia; ${ }^{\text {b Private }}$ \\ Practice, Geneva, Switzerland; ' Division of Orthodontics, University of Geneva, Geneva, Switzerland
}

\section{Significance of the Study}

- This study aimed to qualitatively analyze the content of social media posts to gain an in-depth understanding of patients' experiences surrounding orthognathic surgical treatment. From the posts analyzed, the main themes identified were preoperative engagement, postoperative difficulties, and posttreatment satisfaction. These findings can increase the awareness of clinicians involved in orthognathic surgical treatment, allowing them to better educate and counsel patients.

\section{Keywords}

Health perception · Orthognathic surgery · Orthodontics

\begin{abstract}
Objective: The aim of this qualitative study was to analyze the content of posts on Twitter in order to gain an in-depth understanding of patients' thoughts and experiences surrounding orthognathic surgical treatment. Materials and Methods: Using the Twitter search function, with the keywords "jaw surgery," the 1,000 most recent posts on Twitter with relevance to a combined orthodontic and orthognathic surgical treatment were extracted. After applying relevant inclusion and exclusion criteria, the selected posts were analyzed using thematic analysis by 2 independent investigators. Distinct themes and subthemes were developed. $\boldsymbol{R e}$ sults: A total of 689 posts were analyzed; the 3 main themes
\end{abstract}

identified in relation to orthognathic surgery were preoperative engagement, postoperative difficulties, and posttreatment satisfaction. Twelve subthemes were also identified, expressing issues such as anticipation or apprehension of the surgical procedure, postoperative pain and edema, dietary restrictions and weight loss, paresthesia, depression, and satisfaction with improvements in appearance and selfconfidence. The 6 terms most frequently used in tweets were "recovery," "braces," "swollen," "eat," "liquid diet," and "pain." Conclusions: The findings from the present study can increase the awareness of clinicians involved in the combined orthodontic and orthognathic surgical treatment of patients with dentofacial deformities, allowing them to better educate and counsel their patients throughout the entire treatment process. (c) 2018 The Author(s)

Published by S. Karger AG, Basel

\begin{tabular}{ll}
\hline KARGER & $\begin{array}{l}\text { @ 2018 The Author(s) } \\
\text { Published by S. Karger AG, Basel }\end{array}$ \\
$\begin{array}{l}\text { Openger } \\
\text { E-Mail karger@karger.coms }\end{array}$ \\
www.karger.com/mpp & $\begin{array}{l}\text { This is an Open Access article licensed under the Creative Commons } \\
\text { Attribution-NonCommercial-4.0 International License (CC BY-NC) } \\
\text { (http://www.karger.com/Services/OpenAccessLicense), applicable to } \\
\text { the online version of the article only. Usage and distribution for com- } \\
\text { mercial purposes requires written permission. }\end{array}$
\end{tabular}

Gregory S. Antonarakis

Division of Orthodontics, University of Geneva

19 rue Barthélemy-Menn

$\mathrm{CH}-1205$ Geneva (Switzerland)

E-Mail gregory.antonarakis@unige.ch 


\section{Introduction}

A combined orthodontic and orthognathic surgical approach is a viable treatment option to correct a range of severe dentofacial deformities. Surgical and orthodontic expertise has advanced in an attempt to maximize the quality of the outcome and minimize the risk of posttreatment relapse; however, our understanding of the experiences and perceptions of individuals undergoing this approach to treatment has not yet progressed sufficiently.

It has been shown that research outcomes in orthodontics continue to focus on morphological changes rather than patient-centered outcomes [1]. Professional and objective evaluation of a certain treatment does not fully capture its impact. Patient perceptions are of major importance when evaluating treatment outcomes, especially for elective surgical procedures with associated risks, where the decision-making process needs to be shared between the clinician and the patient. Clinicians should understand the perceptions of the patient, while patients need to be well informed about the procedure, its benefits, and its potential side effects.

Traditional deductive research tools used to investigate patients' perspectives, such as questionnaires, have the disadvantage of narrowing down the themes in question by restricting the emergence of new perspectives. Qualitative methods are thus perhaps more suitable to explore complex phenomena. These methods enable the investigators to comprehend the patient's point of view in an unbiased way [2].

Interview-based qualitative research methods have tried to overcome the limitations of questionnaires and they have recently been used in the field of orthodontics and orthognathic surgery [3]. Face-to-face interviews, nonetheless, also have certain inherent limitations associated with the reluctance of a certain individual to share experiences and perspectives in an uninhibited manner because of interpersonal contact. These limitations have opened the door to other newer sources of data collection, namely from social networking platforms such as Twitter that are growing in popularity, where recent studies have been published in the field of orthodontics and dental pain [4-7]. The use of a qualitative approach to analyze social media content can be used to gain a better insight into the lives of our patients.

Twitter is an online social networking service that enables users to send and read short 140-character messages called "tweets." Registered users can post an unlimited number of tweets, which are by default public and viewable also by unregistered users [8]. As of 2016, Twitter had more than 310 million users, with hundreds of millions of daily tweets and more than a billion search queries performed per day (https://en.wikipedia.org/wiki/ Twitter). A vast amount of health information is publicly available within these tweets [8].

The aim of this study was to qualitatively analyze the content of posts on Twitter in order to gain an in-depth understanding of patients' thoughts and experiences surrounding orthognathic surgical treatment.

\section{Materials and Methods}

\section{Data Source}

We obtained data from a sample of cross-sectional consecutive tweets posted on Twitter relating to orthognathic surgery. Using Twitter's search function, we identified a sample of tweets with relevance to orthognathic surgery by collecting clusters of the 500 most recent tweets from 2016 (up to and including July 31, 2016), searching for the keywords "jaw surgery" or "\#jawsurgery" to identify relevant tweets. Collection of new tweets was carried out in clusters of 500, and this process was repeated until data saturation (no additional information emerging from new data) was reached. The search function of Twitter allows all the key words to be searched simultaneously. The symbol \# denotes a hashtag, which is added to tweets to identify a theme or to categorize tweets, which enables other users to follow related posts with the same hashtag using Twitter's search function.

The data collected included only free, public Twitter posts. We excluded tweets containing hyperlinks (content beginning with "http://" or "https://") and posts that had been posted previously and then reposted by another user (retweets).

\section{Data Extraction}

Identified tweets were entered and compiled in an Excel spreadsheet (version 15.0; Microsoft, Redmond, WA, USA). Tweets were included in the subsequent data analysis if they were related to personal experiences surrounding orthognathic surgery.

Exclusion criteria included the following: professional advertising or promotional posts; third-person posts (concerning jaw surgery of an offspring or sibling, for example); irrelevant, incoherent, or ambiguous posts; jaw surgery relating to trauma, temporomandibular disorders, arthritis, obstructive sleep apnea, or wisdom tooth removal; and posts with an indication that the person was affected by a cleft or craniofacial syndrome.

\section{Content Analysis and Development of Categories}

Analysis of tweet content is hampered by their brevity and by the use of slang, sarcasm, and unconventional forms of written expression [8]. Despite manual coding of Twitter content being slow, it is more accurate when compared with automatic coding since humans are able to process linguistically nuanced text more efficiently than computer-based systems that need extensive training [8]. For this reason, tweets were manually analyzed.

Content analysis of each tweet was carried out using thematic analysis based on the guidelines of Braun and Clarke [9]. Thematic analysis involved reading all of the tweets repeatedly and marking ideas and notes in relation to orthognathic treatment. Two 
investigators, with different clinical backgrounds, independently read and analyzed the tweets. These investigators were blinded to the identity of the Twitter users during content analysis. Tweets were grouped together into topics and then reviewed and refined until meaningful and distinct main themes and subthemes were developed. Areas of disagreement between investigators were resolved by consensus in a case-by-case manner.

\section{Results}

Data from the content of an initial 500 tweets followed by 500 additional tweets were extracted, reaching thematic saturation. Applying the inclusion and exclusion criteria, we excluded 311 tweets, analyzing a final data set of 689 tweets. From these tweets, 3 primary themes were identified, namely preoperative engagement, postoperative difficulties, and posttreatment satisfaction. Twelve subthemes were also identified (Fig. 1).

We entered the final set of tweets into a word frequency calculator to identify the most commonly used words related to the orthognathic surgery experience. The search terms ("jaw" and "surgery") were excluded, as were common English words, words designating time (such as "days" or "tomorrow"), and explicit language. Misspelled

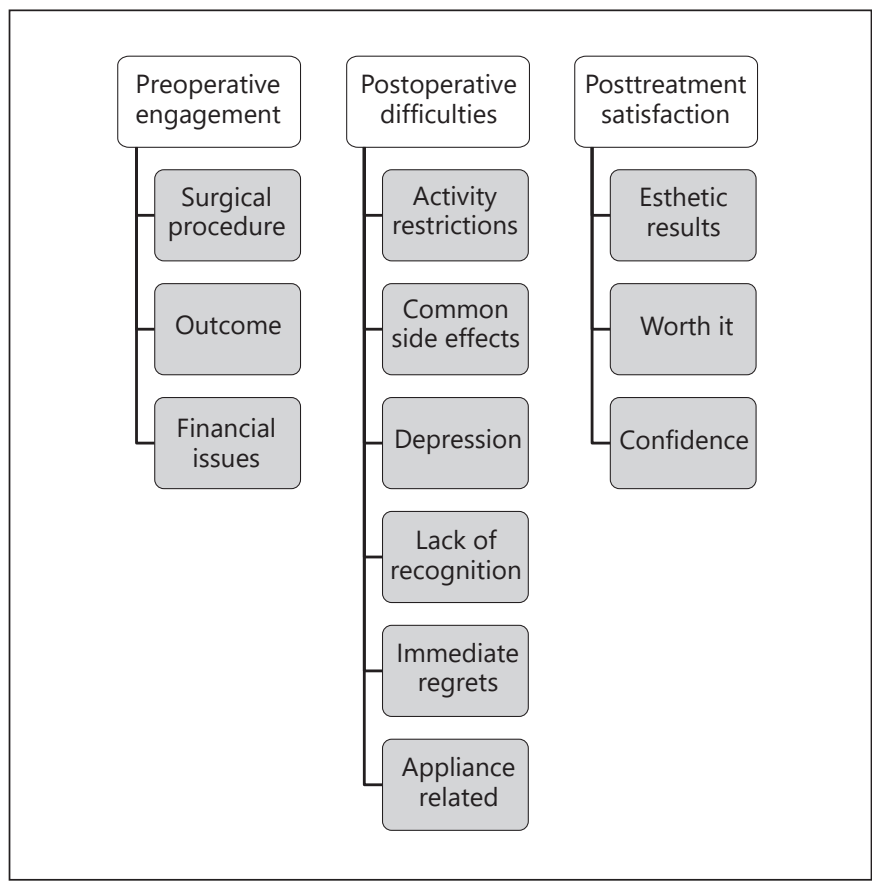

Fig. 1. Themes and subthemes identified concerning the perceptions and values of individuals in relation to a combined orthodontic and orthognathic surgical treatment to correct their dentofacial deformity.

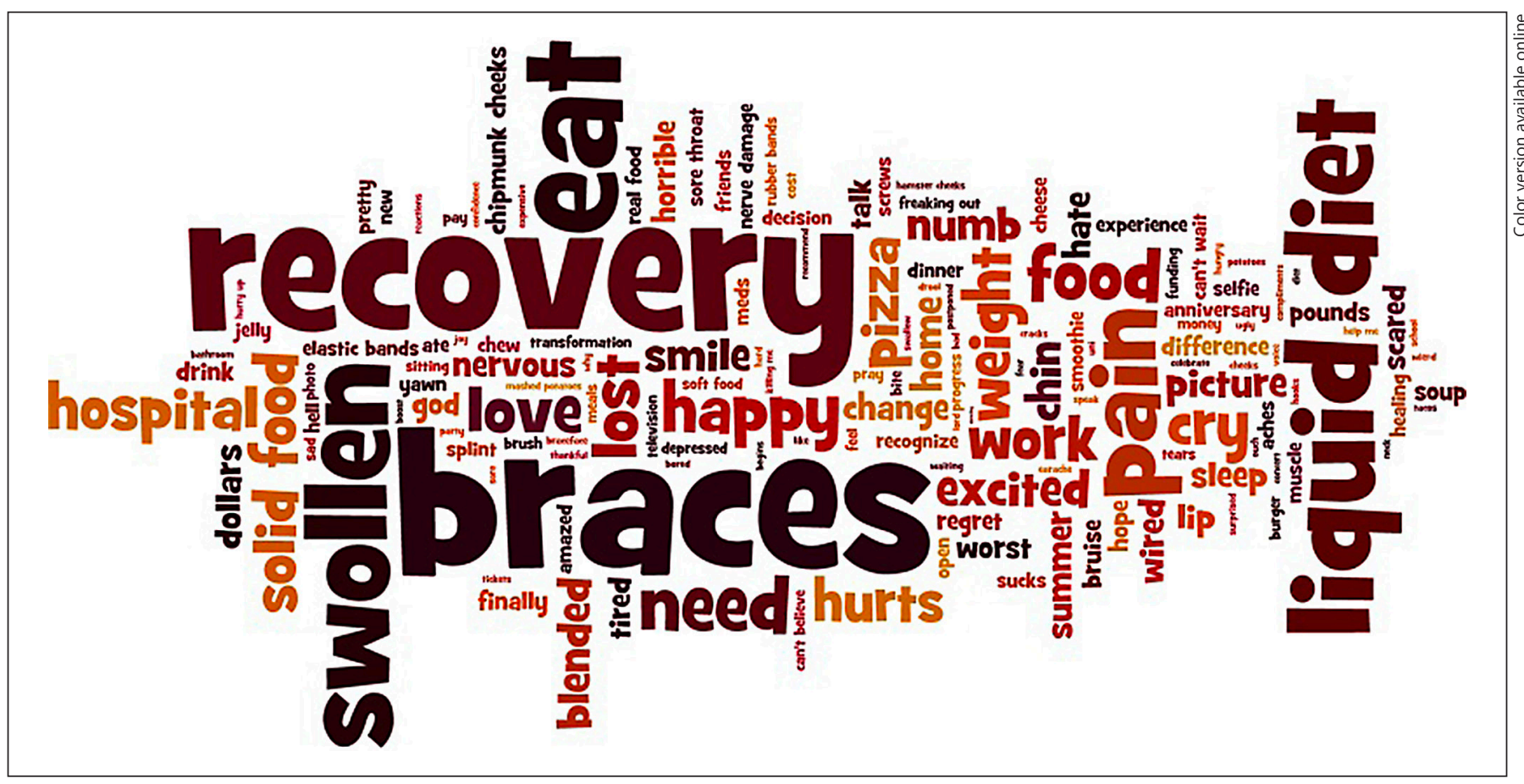

Fig. 2. Word cloud depicting the words used most frequently in the 689 analyzed tweets, excluding the search terms "jaw" and "surgery." Font size correlates with the greater frequency of the word used in the tweets, while word location in the image is random. This image was created using www.wordle.net.
Patient Perspectives of Surgical

Orthodontic Treatment on Social Media
Med Princ Pract

DOI: $10.1159 / 000487904$ 
words were corrected, words appearing in text or abbreviated form were written in full, and one common word was chosen for similar words (e.g., "swollen" and "swelling" were always entered as "swollen" to avoid underrepresentation of words). A word cloud was constructed depicting the words most frequently used in the analyzed tweets (Fig. 2).

Tweets which illustrate each of the identified subthemes are presented in Table 1. Where explicit language is quoted we replaced some letters with "**” to retain the meaning but not cause offence. Where text language is quoted, the abbreviated text word was written out in full for better comprehension. When fully capitalized words were included, capitals were removed. Symbols including emoticons or emojis that are sometimes included in posts to illustrate facial expressions were removed from quotes. Photographs included in tweets were also removed and they were not analyzed.

\section{Preoperative Engagement}

From the content of the tweets, it was evident that many individuals expressed anticipation or apprehension prior to the orthognathic surgical procedure. Tweets ranged from those expressing fear, anxiety, or distress to those expressing mixed feelings, to those expressing hope and eagerness to improve facial esthetics or function. Financial issues were also a concern for some individuals.

\section{Postoperative Difficulties}

A common theme in tweets was the expression of difficulties experienced in relation to postoperative side effects. Side effects such as pain, edema, paresthesia, and fatigue were described, but one often expressed difficulty was dietary restrictions and having to comply with a liquid diet. Some tweets expressed concern over more than one side effect. Due to the postoperative side effects, some individuals admitted signs of depression or went as far as to express regret regarding their surgical procedure. Another interesting theme was difficulty in recognition either by oneself or by others. Tweets sometimes referred to fixed orthodontic appliances, surgical splints, intermaxillary elastics, and surgical hooks. Besides postoperative side effects, appliances also caused discomfort and difficulties. Individuals always seemed eager to finish with and remove their respective appliances.

\section{Posttreatment Satisfaction}

Individuals who tweeted about jaw surgery sometime after the completion of the full orthodontic and orthognathic surgical treatment tended to reflect on the positive changes that this treatment had on them, as opposed to dwelling on the postoperative difficulties. It seems that as more time passes one tends to not focus on the negative but rather on the positive aspects of this treatment. However, some individuals also expressed posttreatment satisfaction very soon after the orthognathic surgical procedure.

\section{Discussion}

The present qualitative study using data available from Twitter identified 3 main themes in relation to orthognathic surgical treatment, namely preoperative engagement, postoperative difficulties, and posttreatment satisfaction. Twelve subthemes were also identified, pertaining to issues such as anticipation or apprehension of the surgical procedure, postoperative pain and edema, dietary restrictions and weight loss, paresthesia, depression, and satisfaction with improvements in appearance and self-confidence. The terms most frequently used in tweets were: "recovery," "braces," "swollen," "eat," "liquid diet," and "pain."

To date, there are no reports on investigations of patients' thoughts and experiences on orthognathic surgery using social networking websites. Larsen and Thygesen [10] performed a questionnaire-based study about treatment satisfaction following orthognathic surgery, using Facebook, but only to recruit patients from an orthognathic surgery Facebook group. No analysis of posts was reported in this study.

\section{Methodological Considerations and Limitations}

The specific use of Twitter in qualitative health research has important advantages. Users often post tweets in real time as an event is happening. This reduces retrospective accounts and recall bias, thus improving data accuracy [4]. Smartphones or tablets can be used to post tweets, which increases their frequency and timeliness [5]. In relation to healthcare, patients use social networking websites to discuss and share problems, concerns, and experiences and to upload pictures, making the use of such data invaluable for healthcare research. Information is universally accessible for various population groups, regardless of factors such as age, sex, education, time, location, nationality, or religion [11]. Lastly, this approach does not disrupt the environment or behavioral patterns of an individual, reducing social desirability and investigator bias $[5,12]$. Patients appear to share their fears and doubts more readily in virtual platforms and are perhaps 
Table 1. Representative tweets illustrating each of the identified subthemes

\begin{tabular}{|c|c|c|}
\hline Theme & Subtheme & Illustrative tweets \\
\hline \multirow[t]{2}{*}{$\begin{array}{l}\text { Preoperative } \\
\text { engagement }\end{array}$} & $\begin{array}{l}\text { Surgical } \\
\text { procedure }\end{array}$ & $\begin{array}{l}\text { "I need this today. I'm trying to be fearless in what is coming my way but I'm still scared \#JawSurgery" } \\
\text { "Getting mentally prepared for jaw surgery! \#Nervous \#Excited \#JawSurgery" }\end{array}$ \\
\hline & Outcome & $\begin{array}{l}\text { "T minus one day. I'll be at the hospital on exactly } 24 \text { hours! Almost one step closer to eating a sandwich that won't fall } \\
\text { apart \#jawsurgery" } \\
\text { "I can’t wait to get jaw surgery \& finally have perfect teeth I don't even care about the recovery I could get it tomorrow" }\end{array}$ \\
\hline \multirow[t]{3}{*}{$\begin{array}{l}\text { Postoperative } \\
\text { difficulties }\end{array}$} & $\begin{array}{l}\text { Activity } \\
\text { restrictions }\end{array}$ & $\begin{array}{l}\text { "My boyfriend is eating my favorite food in front of me and I'm so sad all I want is solid food \#jawsurgery" } \\
\text { "I don't want another smoothie, I want a juicy cheeseburger and fries \#liquiddiet \#jawsurgery" } \\
\text { "When you get two single burgers... because you can't fit a double in your mouth yet \#jawsurgery \#overtwomontheslater" } \\
\text { "Y'all I've lost } 10 \text { pounds on this damn liquid diet because of my horrible jaw surgery, and I'm already small. I look like a } \\
\text { stick now" } \\
\text { "The two best things I do in life (eating and talking) I can't do right now because my jaw surgery and it's the worst" } \\
\text { "So. Tired. Must. Keep. Going \#jawsurgery \#surgery" } \\
\text { "Hey guys. I had a jaw surgery a week and a half ago which has been impeding my ability to exercise" }\end{array}$ \\
\hline & Depression & $\begin{array}{l}\text { "Post-surgical depression is so hard. I wish doctors recognized and had more sympathy for this \#jawsurgery" } \\
\text { "Beyond depressed... Mum bought Ikea meatballs to have for dinner... Took one bite and nearly cried... F*** braces. F*** } \\
\text { double jaw surgery" }\end{array}$ \\
\hline & $\begin{array}{l}\text { Appliance } \\
\text { related }\end{array}$ & $\begin{array}{l}\text { "I get my splint off Wednesday morning! I'm so excited!! \#jawsurgery" } \\
\text { "I can't wait to get home! So glad the braces are off and now I just need the jaw to recover properly! \#brandnewmouth } \\
\text { \#jawsurgery" } \\
\text { "Go through jaw surgery no problem at all, why am I now crying like a baby? Because of the tiny elastic bands I have to } \\
\text { wear" } \\
\text { "Surgical hooks are off. Highlight of my week \#jawsurgery" }\end{array}$ \\
\hline \multirow[t]{3}{*}{$\begin{array}{l}\text { Posttreatment } \\
\text { satisfaction }\end{array}$} & $\begin{array}{l}\text { Esthetic } \\
\text { results }\end{array}$ & $\begin{array}{l}\text { "I love perfect teeth... I guess because I have them. Thanks to braces. And jaw surgery" } \\
\text { "Varda che bel lavoro! Look what great job! (20 days after surgery) \#jawsurgery \#doublejawsurgery..." }\end{array}$ \\
\hline & Worth it & $\begin{array}{l}\text { "A year ago today I looked like a baby hippo but it was all worth it \#jawsurgery \#transformation" } \\
\text { "Wow... It's been } 3 \text { years already. I would absolutely go back and do it again \#jawsurgery" }\end{array}$ \\
\hline & Confidence & $\begin{array}{l}\text { "1 year ago today was my jaw surgery and I honestly couldn't be more happier with the results and the self-confidence } \\
\text { boost" } \\
\text { " } 3 \text { years ago today was the most confidence boosting, life changing day of my life, as I went under the knife for jaw sur- } \\
\text { gery! How time flies" }\end{array}$ \\
\hline
\end{tabular}

Patient Perspectives of Surgical Orthodontic Treatment on Social Media 
more honest and comfortable in sharing positive or negative experiences [13]. It is thus easier to focus on what information patients are seeking, discussing, or sharing of their own accord and away from the clinical environment. This allows investigators to capture a broad range of discriminative themes.

Potential limitations mainly concern bias and the generalizability of the findings. Twitter contains online data, limiting the representation only to online users, which may not necessarily reflect the general population [14]. It has been suggested that online samples are likely to be representative of adolescents or young adults as most have access to the Internet [15], but this may not hold true for other ages. Although Twitter use is fairly well distributed across gender, income, and education levels, users tend to be younger and more racially diverse than the overall population of Internet users [16]. Moreover, it is unclear why patients choose to share their experience through social networking websites, although there may be certain personality characteristics that may cause individuals to do so [4]. Another limitation may be that the posts collected depended on the way the individuals expressed their posts, meaning that some of the posts may have been nonspecific, such as those undergoing orthognathic surgery subsequent to trauma, but not mentioning the trauma in their post. Finally, in the analysis of posts, emoticons were not addressed despite these being a potentially powerful way to communicate feelings. Interpretation of these emoticons, however, is very subjective, hence the decision to remove them from the analysis.

The tweets collected were in the English language since the search terms used were in English. This raises questions about generalizability with respect to differences in geographical location, culture, and language expression. Logically, the great majority of English language tweets would have originated in countries where English is one of the official languages, including mainly the USA, Canada, the UK, Ireland, Australia, and New Zealand but also perhaps other English-speaking countries in the Caribbean, Africa, and Asia. This provides a fairly large geographical distribution.

Bearing this information in mind, it may still be possible to use analogic reasoning to generalize the results of this study to other populations with similar characteristics. The authors did not aim to investigate gender-, age-, geography-, or race-specific differences or differences related to the particular dentofacial deformity or orthognathic surgical procedure, as this would have been impossible based on the data available in the tweets. Given the possible systematic differences between those who do and those who do not use Twitter, one must exercise caution, however, before generalizing results to any particular population.

\section{Putting the Themes Identified into Context with Existing Knowledge}

A recent systematic review looking at pre-, intra-, and postoperative complications in orthognathic surgery identified the following complications: nerve injury/sensitivity alteration, temporomandibular joint disorders or impairment, hemorrhage, auditory tube function and hearing problems, infection, bad split, nonunion of an osteotomy gap, skeletal relapse, septum deviation, bone necrosis, soft tissue injuries, positional vertigo, dental complications, postoperative swelling, and psychological depression [17]. In the present study, no tweets were found referring to several of these complications such as hemorrhage, hearing problems, and infection. Furthermore, no tweets mentioned relapse (either skeletal or dental) as a problem. This may indicate that a small amount of relapse remains unnoticeable or unremarkable for orthognathic surgery patients.

Pain, edema, and difficulty eating were very often alluded to in the tweets analyzed. Based on a previous study using a mail-in questionnaire, many patients reported that pain, edema, and difficulty eating immediately postoperatively were worse than expected [18]. One third of the patients in another study reported that it took them longer to recover from the intervention than anticipated [19].

Individuals tweeted about their satisfaction with the treatment outcome and often stated they were happy with their decision to undergo orthognathic surgery. The satisfaction of participants following orthodontic and orthognathic surgery has been found to be high from previous studies [20,21]. In addition, orthognathic patients are more likely to be satisfied when they are provided with more information about discomfort and surgical risks [22] and forewarned about negative events such as pain, numbness, and swelling [20,23]. The reason why patients seek out orthognathic surgery, and whether their expectations were realistic, may impact their satisfaction. It has been found that correction of the bite or functional disability may be a more common reason to seek a surgical evaluation than improvement of appearance [24]. Concerning self-confidence, a systematic review found improvement after combined orthodontic and orthognathic surgical treatment [24]. A more recent questionnairebased study found that an important posttreatment outcome is an improved self-concept, which also improves the posttreatment satisfaction [19]. 
An interesting theme expressed in some of the tweets was the difficulty dealing with the change in appearance, especially relating to self-recognition or recognition from others. Friends and family can have problems getting used to or accepting the new appearance of the patient [25], and this can be problematic and discouraging to patients [3]. It has been observed, however, that the opinions of friends and family affect patient satisfaction, and those who receive comfort from their entourage present a better psychological health status [26-28].

Dos Santos et al. [29] carried out a study looking into the perception of orthognathic surgery patients on postoperative care, using a focus group; through content analysis they identified problems related to a long recovery time, nutrition, oral hygiene, and relief of postoperative signs and symptoms. Themes involving self-image and satisfaction levels were also recognized. The statements of the participants clearly showed that, in order for the information to be internalized, patients should not only be informed but they also need to understand what the health professional says, in order to help them to cope with experienced adversities in the postoperative period [29].

Sadat-Marashi et al. [3] used a grounded theory approach with interviews of individuals having undergone a combined orthodontic and orthognathic surgical treatment and identified the following themes: satisfaction with the treatment outcome despite the difficult experience, fears caused by swelling and complications, pain caused by orthodontic treatment, difficulty eating, shock for family and close friends, experiences shared by those already having undergone a similar treatment, and smiling with self-confidence. These were similar to those found in the current study, apart from the desire to speak to someone having already undergone a similar treatment. This may be because the Internet is a medium where experiences are shared, thus eliminating the need to speak to someone face-to-face. This slightly impersonal form of communication can be interesting as patients may actually share more information or more accurate information than they would in a face-to-face setting.

Few studies exist exploring how individuals use the Internet to find or share information relating to orthognathic surgery. Bhamrah et al. [12] found that patients undergoing orthognathic surgery look to the Internet for supplemental information regarding their treatment. The authors identified the following themes from Internet discussion forums: reasons for undergoing orthognathic treatment; presurgery and postsurgery treatment stages including orthodontics; surgery including postsurgical

Patient Perspectives of Surgical

Orthodontic Treatment on Social Media complications and difficulties; expected and actual end of treatment changes; and seeking and sharing information. There were limited discussions regarding the actual surgical procedure and the general anesthetic. This was also the case in the present study where individuals did not tweet about the surgical procedure itself or about specific fears with having to undergo general anesthesia. From a clinician's perspective, the focus is on planning, execution, and evaluation of the treatment whereas from the patient's perspective one of the most important time points is when they wake from the anesthesia. From that moment their focus is on pain, swelling, nutrition, and coping with their appliances. If we look at the treatment pathway from the patients' perspective, the results of the study are more clearly understood.

Sousa and Turrini [13] and Do Rego Barros et al. [30] analyzed comments from blogs and virtual communities related to orthognathic surgery to gain a greater understanding of what patients are discussing. Content analysis identified several themes including concerns and fears during the perioperative period (such as costs, returning to daily activities, and anesthesia), postoperative recovery (such as difficulty in eating and oral hygiene, paresthesia, respiration, facial edema, pain, and voice changes), and changes in facial aesthetics (body image and self-esteem). However, their analyses were limited to expectations or concerns regarding orthognathic surgery and did not include the entire orthognathic treatment journey. In addition, the research only focused on discussions in the Portuguese language.

Medicolegal related issues have influenced many areas of clinical practice, particularly for surgery and the informed consent process. More time is often spent discussing potential serious complications while less time is dedicated to addressing coping strategies for side effects that nearly every patient will experience. The recognition and categorization of perioperative issues identified in the present study will help clinicians to better plan and allocate their time for patient care.

\section{Seeking Online Support and Unmet Needs}

The need to seek information online could suggest a possible gap in the provision of information by professionals. Internet discussion forums allow patients to interact with their peers and offer and seek advice, support, and information [31]. Healthcare providers are often focused on providing information that enables patients to consent to treatment in an informed manner, but they can occasionally fail to give the counseling and practical advice that patients require to improve their experience 
[12]. Patients must also be made aware of the limitations in seeking counseling online and guided by specialists to consult good quality reliable websites, especially since it has been found that the majority of websites on orthognathic surgery fall below a reasonable standard [32]. By directing patients to validated websites, clinicians can ensure that patients find appropriate information; however, continuous development of websites relating to orthognathic surgery with regular updates is required [32].

Another reason that patients seek information online may be that, even though information has been given, they want reinforcement and real-life experiences of peers, and the Internet is the easiest place to find these [12]. The search for information on the Internet enables an interchange of experiences among patients with similar health problems and provides support, emotional encouragement, and reassurance from peers.

Traditionally patients may seek further information or opinions about a procedure from a friend or acquaintance who has been through a similar experience. Often there is a disproportionately high value placed on these opinions even though they are not founded on a strong scientific basis. With access to online forums, the ability to identify someone who has been through a similar surgical procedure is easier than ever before. There is valid concern that such forums may disseminate inaccurate information. As the popularity of online forums continues to grow, there is a need for clinicians to work with social media and develop ways to improve the quality of the information provided to patients in those formats.

\section{Clinical Implications}

For surgeons, orthognathic surgery is a routine procedure, but for patients it is a big, unknown, time-consuming, and risky treatment representing an important burden of care [33]. A better understanding of how patients view and experience the impact of combined orthodontic and orthognathic surgical treatment on their lives is pivotal to counseling patients and families adequately. This can provide an easier and less eventful recovery. A critical component of good patient care is anticipating problems and preparing patients for them. Patient satisfaction and compliance are greatly increased if they are prepared in advance rather than addressing problems once they arise.

During a medical appointment, patients may feel embarrassed to ask trivial questions and prefer to turn to the Internet for answers [13]. The information shared by patients on the Internet about their care or experiences acts as an indicator of the possible weaknesses in the communication schemes and instructions provided by health- care professionals, suggesting that the patient did not receive or understand the instructions provided. Patients may post information that they consider to be essential but professionals consider too basic to be of concern, and it is therefore vital to understand what information patients require rather than what healthcare professionals think the patients should know [13].

Counseling and psychological support may be more useful than is perhaps thought. One suggestion may be to provide this support during the decision-making process, immediately preoperatively, immediately postoperatively, and perhaps 1 year after the surgery. This would help patients to get better informed when deciding whether to undergo this treatment modality, get prepared for the surgery, be better able to cope with the postoperative side effects, and finally come to terms with their new appearance. Whether this counseling and support comes from a psychologist, a psychiatrist, a surgeon, an orthodontist, or a trained nurse is open for discussion and will depend on many local factors. Do Rego Barros et al. [30] propose that nurses can play an important role as educators through spoken or written communication about concerns regarding orthognathic surgery. Properly oriented patients feel more prepared for surgical procedures, cope better with difficulties experienced postoperatively, and ultimately manifest greater satisfaction with treatment outcomes [29].

\section{Conclusions}

The findings from the present study provide information regarding patients' experiences with orthognathic surgery as shared publicly on Twitter. This knowledge can help clinicians involved in orthodontic and orthognathic surgical treatments of patients with dentofacial deformities to become more aware and consequently better qualified to adequately advise and counsel their patients throughout the treatment process. 


\section{References}

$>1$ Tsichlaki A, O’Brien K: Do orthodontic research outcomes reflect patient values? A systematic review of randomized controlled trials involving children. Am J Orthod Dentofacial Orthop 2014;146:279-285.

-2 Hallberg LR: The "core category" of grounded theory: making constant comparisons. Int J Qual Stud Health Well-being 2006;1:141148.

-3 Sadat-Marashi Z, Scolozzi P, Antonarakis GS: Perceptions of young adults having undergone combined orthodontic and orthognathic surgical treatment: a grounded theory approach. J Oral Maxillofac Surg 2015;73: 2391-2398.

4 Heaivilin N, Gerbert B, Page JE, et al: Public health surveillance of dental pain via Twitter. J Dent Res 2011;90:1047-1051.

5 Henzell MR, Knight AM, Morgaine KC, et al: A qualitative analysis of orthodontic-related posts on Twitter. Angle Orthod 2014;84:203207.

$\checkmark 6$ Al-Moghrabi S, Johal A, Fleming PS: What people are tweeting about orthodontic retention? A cross-sectional content analysis. Am J Orthod Dentofacial Orthop 2017;152:516522.

7 Noll D, Mahon B, Shroff B, et al: Twitter analysis of the orthodontic patient experience with braces vs. Invisalign. Angle Orthod 2017; 84:377-383.

-8 Kim AE, Hansen HM, Murphy J, et al: Methodological considerations in analyzing Twitter data. J Natl Cancer Inst Monogr 2013, 2013:140-146.

9 Braun V, Clarke V: Using thematic analysis in psychology. Qual Res Psychol 2006;3:77-101.

10 Larsen MK, Thygesen TH: Orthognathic surgery: outcome in a Facebook group. J Craniofac Surg 2016;27:350-355.

11 Moorhead SA, Hazlett DE, Harrison L, et al: A new dimension of health care: systematic review of the uses, benefits, and limitations of social media for health communication. Med Internet Res 2013;15:e85.

Patient Perspectives of Surgical Orthodontic Treatment on Social Media
12 Bhamrah G, Ahmad S, NiMhurchadha S: Internet discussion forums, an information and support resource for orthognathic patients. Am J Orthod Dentofacial Orthop 2015;147: 89-96.

13 Sousa CS, Turrini RN: Creating and validating educational material for patients undergoing orthognathic surgery. Asian Nurs Res (Korean Soc Nurs Sci) 2012;6:166-172.

4 Eke PI: Using social media for research and public health surveillance. J Dent Res 2011;90. 1045-1046.

15 Moreno MA, Fost NC, Christakis DA: Research ethics in the MySpace era. Pediatrics 2004;121:157-161.

16 Smith A, Brenner J: Twitter use 2012: Pew Internet and American Life Project 2012. http:// www.pewinternet.org/2012/05/31/twitteruse-2012/.

17 Jędrzejewski M, Smektała T, Sporniak-Tutak K, et al: Preoperative, intraoperative, and postoperative complications in orthognathic surgery: a systematic review. Clin Oral Investig 2015;19:969-977.

18 Williams RW, Travess HC, Williams AC: Patients' experiences after undergoing orthognathic surgery at NHS hospitals in the south west of England. Br J Oral Maxillofac Surg 2004;42:419-431.

19 Oland J, Jensen J, Elklit A, et al: Motives for surgical-orthodontic treatment and effect of treatment on psychosocial well-being and satisfaction: a prospective study of 118 patients. J Oral Maxillofac Surg 2011;69:104-113.

20 Nurminen L, Pietilä T, Vinkka-Puhakka H: Motivation for and satisfaction with orthodontic-surgical treatment: a retrospective study of 28 patients. Eur J Orthod 1999;21: 79-87.

21 Song YL, Yap AU: Orthognathic treatment of dentofacial disharmonies: its impact on temporomandibular disorders, quality of life, and psychosocial wellness. Cranio 2017;35:52-57.

22 AlKharafi L, AlHajery D, Andersson L: Orthognathic surgery: pretreatment information and patient satisfaction. Med Princ Pract $2014 ; 23: 218-224$
23 Cunningham SJ, Crean SJ, Hunt NP, et al: Preparation, perceptions, and problems: a long-term follow-up study of orthognathic surgery. Int J Adult Orthodon Orthognath Surg 1996;11:41-47.

24 Proothi M, Drew SJ, Sachs SA: Motivating factors for patients undergoing orthognathic surgery evaluation. J Oral Maxillofac Surg 2010;68:1555-1559.

25 Hunt OT, Johnston CD, Hepper PG, et al: The psychosocial impact of orthognathic surgery: a systematic review. Am J Orthod Dentofacial Orthop 2001;120:490-497.

26 Rustemeyer J, Eke Z, Bremerich A: Perception of improvement after orthognathic surgery: the important variables affecting patient satisfaction. Oral Maxillofac Surg 2010;14:155162

27 Chen B, Zhang ZK, Wang X: Factors influencing postoperative satisfaction of orthognathic surgery patients. Int J Adult Orthodon Orthognath Surg 2002;17:217-222.

28 Holman AR, Brumer S, Ware WH, et al: The impact of interpersonal support on patient satisfaction with orthognathic surgery. J Oral Maxillofac Surg 1995;53:1289-1297.

29 Dos Santos MR, Sousa CS, Turrini RN: Perception of orthognathic surgery patients on postoperative care. Rev Esc Enferm USP 2012; 46:78-85.

30 Do Rego Barros B, Sousa CS, Turrini RN: Knowledge of Internet-using patients about the perioperative period of orthognathic surgery. J Nurs Educ Pract 2013;3:93-102.

31 Armstrong N, Powell J: Patient perspectives on health advice posted on Internet discussion boards: a qualitative study. Health Expect 2009; $12: 313-320$

32 Aldairy T, Laverick S, McIntyre GT: Orthognathic surgery: is patient information on the Internet valid? Eur J Orthod 2012;34:466469

33 Hagensli N, Stenvik A, Espeland L: Patient of fered orthognathic surgery: why do many refrain from treatment? J Craniomaxillofac Surg 2014;32:e296-e300. 